\title{
Identification of Industrial Furnace Temperature for Sintering Process in Nuclear Fuel Fabrication Using NARX Neural Networks
}

\author{
Dede Sutarya $^{1,2}$ and Benyamin Kusumoputro ${ }^{1}$ \\ ${ }^{1}$ Department of Electrical Engineering, University of Indonesia, Kampus Baru UI, Depok 16424, Indonesia \\ ${ }^{2}$ Center for Nuclear Fuel Technology, National Nuclear Energy Agency, Kawasan PUSPIPTEK, Tangerang 15314, Indonesia \\ Correspondence should be addressed to Dede Sutarya; dede.sutarya@ui.ac.id
}

Received 30 August 2013; Accepted 5 January 2014; Published 3 April 2014

Academic Editor: Alejandro Clausse

Copyright (c) 2014 D. Sutarya and B. Kusumoputro. This is an open access article distributed under the Creative Commons Attribution License, which permits unrestricted use, distribution, and reproduction in any medium, provided the original work is properly cited.

\begin{abstract}
Nonlinear system identification is becoming an important tool which can be used to improve control performance and achieve robust fault-tolerant behavior. Among the different nonlinear identification techniques, methods based on neural network model are gradually becoming established not only in the academia, but also in industrial application. An identification scheme of nonlinear systems for sintering furnace temperature in nuclear fuel fabrication using neural network autoregressive with exogenous inputs (NNARX) model investigated in this paper. The main contribution of this paper is to identify the appropriate model and structure to be applied in control temperature in the sintering process in nuclear fuel fabrication, that is, a nonlinear dynamical system. Satisfactory agreement between identified and experimental data is found with normalized sum square error $1.9 e-03$ for heating step and $6.3859 e-08$ for soaking step. That result shows the model successfully predict the evolution of the temperature in the furnace.
\end{abstract}

\section{Introduction}

Temperature controllers must set the temperature very accurately in order to meet the needs of technological processes. So, when we design a controller for an electric furnace, precision is extremely important. To provide an exact model of the furnace for this purpose, we identify the system from measured data. Many systems are not amenable to conventional modeling approaches due to the lack of precise, formal knowledge about the system, due to strongly nonlinear behavior, high degree uncertainty, or time-varying characteristics. Sintering furnace is a nonlinear dynamic system and control problems are challenging in the industry. Most dynamical systems can be better represented by nonlinear models, which are able to describe the global behavior of a system over the whole operating range. The behavior of most nonlinear dynamical systems has made the use of artificial neural networks (ANNs) for identification task. The application of ANNs to modeling and control nonlinear process has been intensively studied in recent years [1]. In addition, all numerous studies have shown that multilayer perceptrons (MLPs) neural network is very good choice for nonlinear system identification [2]. Basically there are four types of basic learning rules: Competitive Learning, Error Correction Learning, Hebbian Learning, and Boltzmann Learning [3]. Among all the training algorithms the most popular choice is Back Propagation (BP) which is followed by the error correction learning rule. The neural network autoregressive model with exogenous input has been used for the identification of temperature control, such as modeling greenhouse temperature [4], modeling and identification of heat exchanger [5], and thermal dynamic identification of a pulsating heat pipe [6], and for the other system identification applications which are shown in the literature [7-10].

In this paper, multilayer perceptron (MLP) neural network with nonlinear autoregressive with exogenous input (NNARX) is used to identify a sintering furnace temperature in nuclear fuel fabrication process. Section 2 explains the 


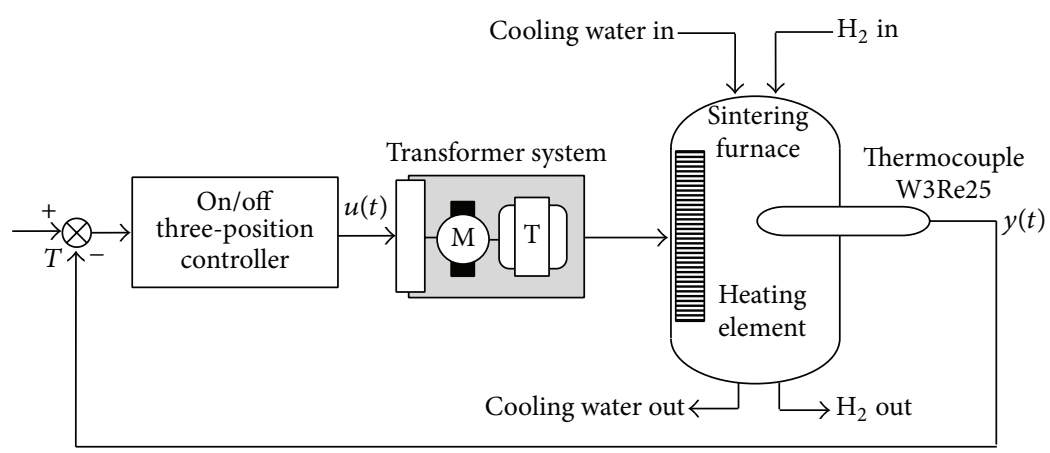

FIGURE 1: Sintering furnace temperature control system.

sintering furnace characteristic and method of data collection process. Identification of the sintering furnace temperature using neural network is explained in Section 3. Simulation results and discussion are presented in Section 4.

\section{Sintering Furnace and Data Collection Methods}

2.1. Sintering Furnace. Sintering is a heat treatment applied to a powder compact in order to impart strength and integrity. The temperature used for sintering is below the melting point of the major constituent of the powder metallurgy material. Control for the heating rate, time, temperature, and atmosphere is required for reproducible results.

The sintering process of uranium dioxide pellets was carried in a furnace with three steps, namely, constant heating rate, soaking temperature, and cooling steps. Heating rate, soaking temperature, and soaking time were adjusted as a needed while cooling stage is done naturally.

Experimental results are obtained from industrial heattreating sintering furnace with pure hydrogen atmosphere. Figure 1 shows the embedded control loop in the sintering furnace. The input to the furnace is a three-position on-off control signal that switches the heating element to the main supply through an autotransformer. The output is the temperature feedback into the three-position on-off controller. The furnace has a maximum capacity of $50 \mathrm{~kg}$ of uranium dioxide pellets with a maximum temperature of $1800^{\circ} \mathrm{C}$ under the hydrogen atmosphere. In the maximum operating conditions a furnace will require the electrical power of about $80 \mathrm{KW}$. The heating rate in the furnace can be adjusted to a maximum of $350^{\circ} \mathrm{C} / \mathrm{h}$ and sintering time can be adjusted as needed, and use conventional cooling method (water-jacket cooling system) [12].

2.2. Data Collection Methods. The interfacing of analytical measurement instrumentation to personal computers (PC) for the purpose of online data acquisition has now become standard practice in the modern laboratory. To eliminate the timing uncertainty from temperature measurements, a new data acquisition program has been implemented, specific for the furnace. As shown in Figure 2 the program running on the PC communicates with the controller. The control program

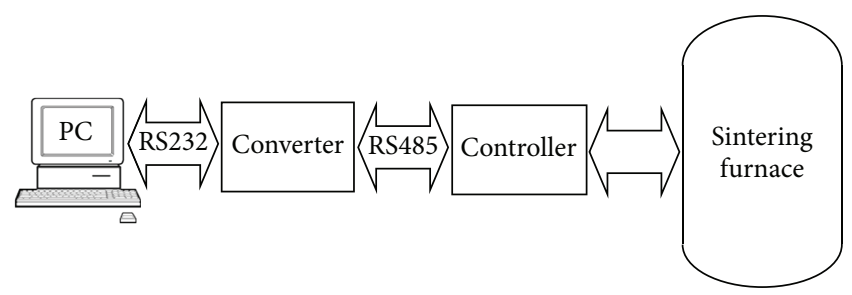

FIgURE 2: A setup temperature measurement inside sintering furnace.

is entered into the controller before the measurement starts. During measurement, the data acquisition system (DAQ) program polls the controller for the measured temperature and the current input signal. The control loop is not closed in the measurement setup. The controller simply directs the process and does not control it. Data collection was conducted during two cycles of the sintering process about 48 hours to obtain training and testing data set separately.

A total of 1600 data samples are taken from heating and sintering phase which describes the behavior of temperature in sintering furnace. The data set is then divided into two parts, 50 percent of the data set for training the neural networks and the rest of the validation objectives. Figure 3 shows the training and testing data set.

\section{System Identification with NNARX Model}

The identification system is the science of how to construct mathematical models of dynamic systems with observation of input and output data. The first step in the identification process has designed a suitable experiment which best brings out the acquired data containing maximum information regarding the process [13]. The collected data are subjected to some preprocessing technique in order to remove the effect of undesired noise and imperfections. Then, a set of candidate models is obtained. The next step is to verify the quality of the developed model. If the model meets the chosen criteria which reflect the intended use of the model, the model is accepted; otherwise, it is rejected and another model is created. This procedure is repeated until a satisfactory model is created. 


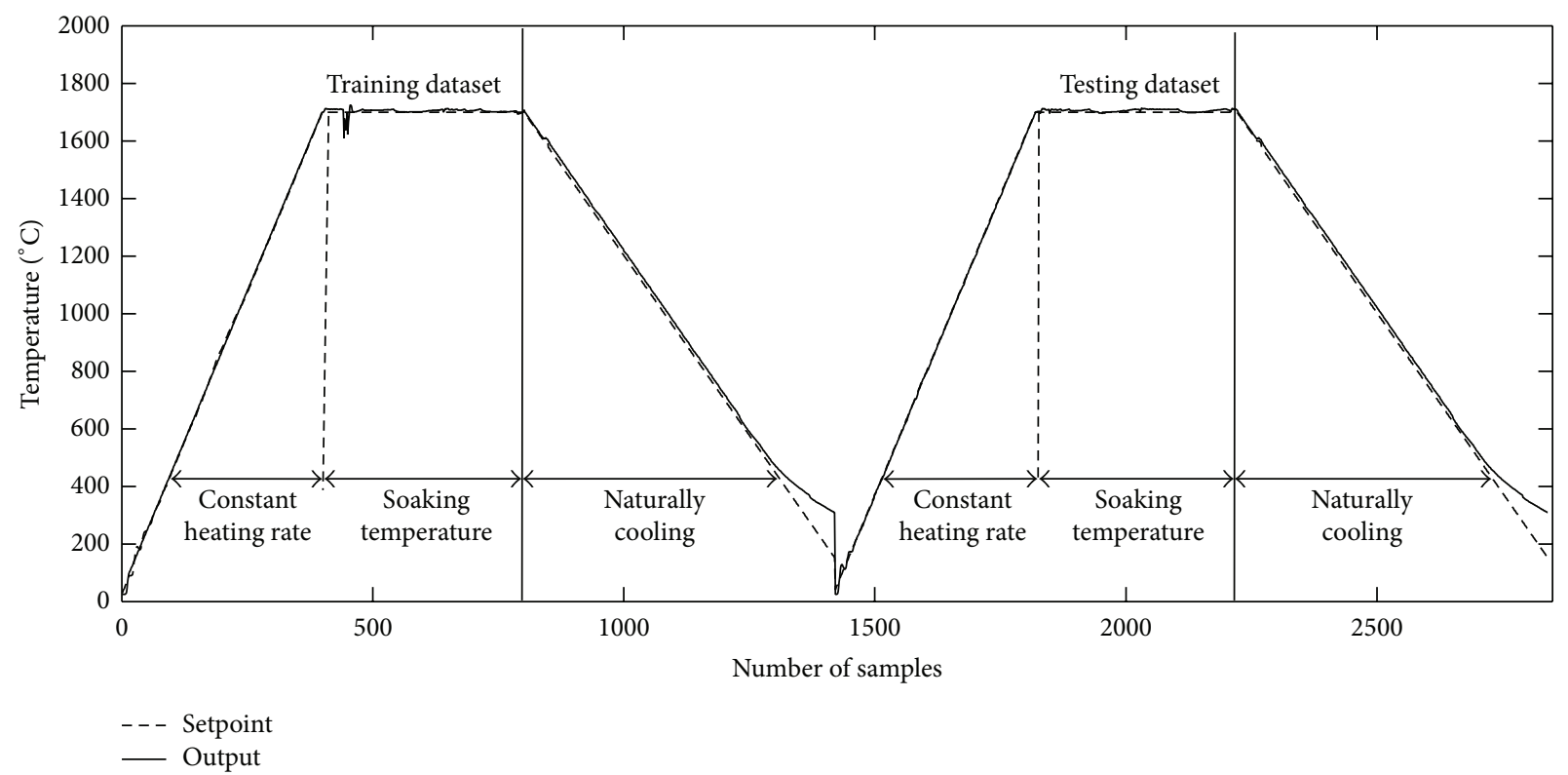

FIgURE 3: Training and testing dataset.

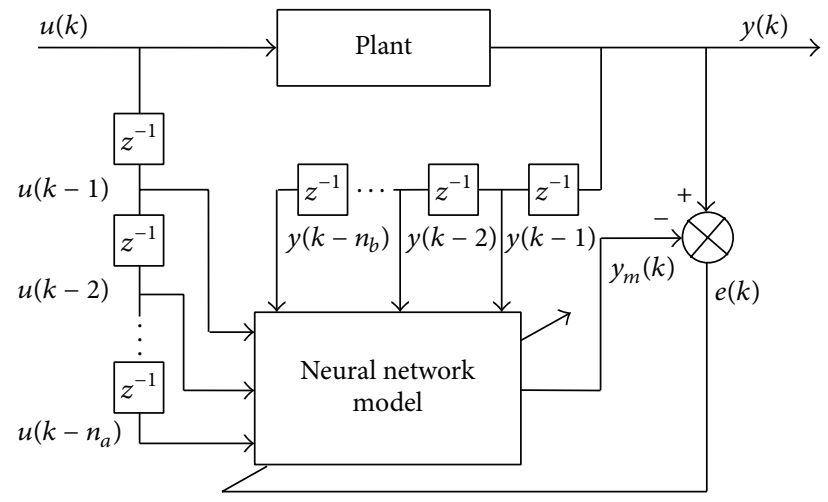

FIGURE 4: General blocks scheme of the NARX model.

Various methods have been developed in the literature for nonlinear system identification. These methods use a parameterized model. The parameters are updated to minimize an output identification error. A nonlinear dynamical system with input $u$ and output $y$ can be explained by the model as

$$
y_{m}(k)=f_{m}(\varphi(k), \theta),
$$

where $y_{m}(k)$ is the output of the model, $\varphi(k)$ is the regression vector, and $\theta$ is the parameter vector. Depending on the choice of the regressors $\varphi(k)$, for NARX (nonlinear autoregressive with exogenous inputs) model can be derived:

$$
\begin{array}{r}
\varphi(k)=\left[u(k-1), u(k-2), \ldots, u\left(k-n_{a}\right), \ldots\right. \\
\left.y(k-1), y(k-2), \ldots, y\left(k-n_{b}\right)\right],
\end{array}
$$

where $n_{a}$ denotes the maximum lag of input and $n_{b}$ is the maximum lag of output. Figure 4 shows the representation of nonlinear systems for NARX models.
Neural networks can be classified as feed-forward and recurrent network. The two-layer feed-forward neural network with sigmoid activation function in the hidden layer and linear activation function in the output layer has the ability to estimate the nonlinear functions if the number of the neurons in the hidden layer is large enough. Figure 5 displays the feed-forward neural network used in this paper.

The input vector to the neural network is defined as

$$
\begin{array}{r}
I^{T}(k)=\left[u(k-1), u(k-2), \ldots, u\left(k-n_{a}\right), \ldots\right. \\
\left.y(k-1), y(k-2), \ldots, y\left(k-n_{b}\right)\right] .
\end{array}
$$

The inputs $u(k-1), u(k-2), \ldots, u\left(k-n_{a}\right)$, and $y(k-1), y(k-$ $2), \ldots, y\left(k-n_{b}\right)$ are multiplied by weights $w_{u i j}$ and $w_{y i j}$, respectively, and summed at each hidden node. Then the sum of the product of the weights and input at a node activated by a sigmoid function. Thus, the output $y_{m}(k)$ in the linear output node can be calculated from its inputs as follows:

$$
\begin{aligned}
y_{m}(k)= & \sum_{i=1}^{N_{h}} \omega_{i} \\
& \times \frac{1}{1+e^{-\left(\sum_{j=1}^{n_{a}} u(k-j) w_{u i j}+\sum_{j=1}^{n_{b}} y(k-j) w_{y i j}+b_{i}\right)}}+b,
\end{aligned}
$$

where $n_{a}+n_{b}$ is the number of inputs, $N_{h}$ is the number of hidden neuron, $w_{u i j}$ is the first layer weight between the input $u(k-j)$ and the $i$ th hidden neuron, $w_{y i j}$ is the first layer weight between the input $y(k-j)$ and the $i$ th hidden neuron, $w_{i}$ is the second layer weight between $i$ th hidden neuron and output neuron, $b_{i}$ is a biased weight for the $i$ th hidden neuron, and $b$ is a biased weight for the output neuron. The difference between the output of the plant $y(k)$ and the output of the model $y_{m}(k)$ is called the prediction error:

$$
e(k)=y(k)-y_{m}(k)
$$




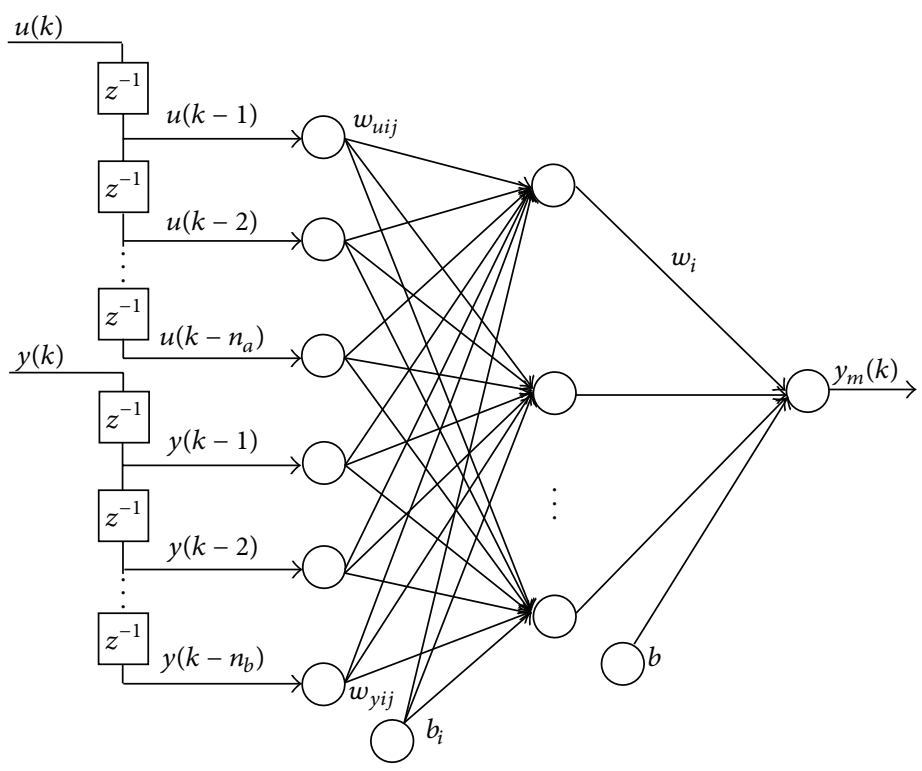

FIGURE 5: Feed-forward neural network structure [11].

This error is used to adjust the weight and biases in the network via the minimization of the following function:

$$
\varepsilon=\frac{1}{2}\left[y(k)-y_{m}(k)\right]^{2}
$$

\section{Result and Discussion}

4.1. Model Order Selection. Empirical models for the description of physical phenomena can be obtained from openloop or closed-loop plant data $[13,14]$. There has been considerable interest in obtaining models from data collected under closed-loop operation [15-18]. Closed-loop identification requires less monitoring of the plant, but it correlates the noise and input signal. Appropriate specification of the model order for system identification is required to represent the physical process adequately.

To establish a suitable NARX model order for a particular system, neural networks of increasing model order can be trained and their performances on the training data compared using the loss function. The function is expressed by the following equation:

$$
\mathrm{LF}=\frac{1}{N} \sum_{i=1}^{N} \varepsilon^{2}(t),
$$

where $N$ is the data length. A model shows that a lower loss function (LF) is not necessarily the best choice because it is a trade-off between model complexity and accuracy. A small decrease in the loss function can be rejected if it is at the expense of enlarging the model size. Thus, the decision procedure is not enough to choose the model using loss function. The difficult trade-off between model accuracy and complexity can be clarified by using model parsimony indices from linear estimation theory, such as Aikeke's Information Criterion (AIC), Bayesian Information Criterion (BIC), and Rissanen's Minimum Description Length (MDL). Validation step is necessary so that it is possible to distinguish the model describing correctly the dynamic behavior of the process. The AIC, BIC, and MDL are defined as follows:

$$
\begin{gathered}
\mathrm{AIC}=\ln \left(\frac{N}{2} \mathrm{LF}\right)+\frac{2 n_{w}}{N} \\
\mathrm{BIC}=\ln \left(\frac{N}{2} \mathrm{LF}\right)+\frac{n_{w} \ln (N)}{N} \\
\mathrm{MDL}=\ln \left(\frac{N}{2} \mathrm{LF}\right)+\frac{2 n_{w} \ln (N)}{N},
\end{gathered}
$$

where $n_{w}$ is the number of model parameters (weight in a neural network).

The process models are obtained by using trial and error method to the NARX model structure with delay operators $\left(n_{k}\right)$ for the measurement $y$ and input $u$. Table 1 shows that the structure of the NARX models with $n_{a}=2, n_{b}=1, n_{k}=$ 7 , and number of hidden neurons 15 has the lowest value of the loss function of the constant heating rate step, while that for soaking temperature step the NARX model structure with $n_{a}=2, n_{b}=3, n_{k}=8$, and number of hidden neurons 5 has the lowest value of the loss function as shown in Table 2.

However, the structure is not necessarily the best option. Therefore, AIC, BIC, and MDL were calculated to support the selection of the most satisfying model structure for the system to be identified. Hence, the AIC, BIC, and MDL are a weighted function of the loss function which penalizes for a reduction in the prediction error at the expense of increasing model complexity (e.g., model order and number of parameters). However, in practice, engineering judgment may need to be carried out. In this case, the criteria AIC, BIC, 
TABLE 1: Loss function (LF) and AIC, BIC, and MDL criterion obtained by trial and error with difference NARX neural network structure and number of hidden layers for constant heating rate step.

\begin{tabular}{|c|c|c|c|c|c|}
\hline \multirow{2}{*}{ Hidden node number } & \multirow{2}{*}{ Model order $\left[\begin{array}{lll}n_{a} & n_{b} & n_{k}\end{array}\right]$} & \multirow{2}{*}{$\mathrm{LF}$} & \multicolumn{3}{|c|}{ Criterion } \\
\hline & & & AIC & BIC & MDL \\
\hline \multirow{5}{*}{5} & {$\left[\begin{array}{lll}2 & 1 & 9\end{array}\right]$} & $2.6 e-05$ & -5.2402 & -5.2103 & -5.1653 \\
\hline & {$\left[\begin{array}{lll}2 & 2 & 2\end{array}\right]$} & $6.5 e-06$ & -6.6331 & -6.5931 & -6.5332 \\
\hline & {$\left[\begin{array}{lll}2 & 3 & 5\end{array}\right]$} & $2.0 e-05$ & -5.5014 & -5.4515 & -5.3766 \\
\hline & {$\left[\begin{array}{lll}3 & 1 & 1\end{array}\right]$} & $6.2 e-06$ & -6.6774 & -6.6375 & -6.5776 \\
\hline & {$\left[\begin{array}{lll}3 & 2 & 8\end{array}\right]$} & $8.6 e-06$ & -6.3369 & -6.2870 & -6.2121 \\
\hline \multirow{5}{*}{10} & {$\left[\begin{array}{lll}2 & 1 & 9\end{array}\right]$} & $3.3 e-05$ & -5.0209 & -4.9910 & -4.9460 \\
\hline & {$\left[\begin{array}{lll}2 & 2 & 1\end{array}\right]$} & $1.2 e-04$ & -3.7181 & -3.6781 & -3.6182 \\
\hline & {$\left[\begin{array}{lll}2 & 3 & 4\end{array}\right]$} & $5.3 e-06$ & -6.8339 & -6.7840 & -6.7091 \\
\hline & {$\left[\begin{array}{lll}3 & 1 & 6\end{array}\right]$} & $3.3 e-05$ & -5.0067 & -4.9668 & -4.9069 \\
\hline & {$\left[\begin{array}{lll}3 & 2 & 7\end{array}\right]$} & $1.5 e-05$ & -5.7774 & -5.7276 & -5.6527 \\
\hline \multirow{5}{*}{15} & {$\left[\begin{array}{lll}2 & 1 & 7\end{array}\right]$} & $3.4 e-06$ & -7.2667 & -7.2367 & -7.1918 \\
\hline & {$\left[\begin{array}{lll}2 & 2 & 6\end{array}\right]$} & $7.9 e-06$ & -6.4328 & -6.3929 & -6.3330 \\
\hline & {$\left[\begin{array}{lll}2 & 3 & 9\end{array}\right]$} & $8.5 e-02$ & 2.8593 & 2.9092 & 2.9841 \\
\hline & {$\left[\begin{array}{lll}3 & 1 & 7\end{array}\right]$} & $3.3 e-03$ & -0.3955 & -0.3556 & -0.2956 \\
\hline & {$\left[\begin{array}{lll}3 & 3 & 7\end{array}\right]$} & $3.3 e-05$ & -4.9836 & -4.9337 & -4.8588 \\
\hline
\end{tabular}

TABLE 2: Loss function (LF) and AIC, BIC, and MDL criterion obtained by trial and error with difference NARX neural network structure and number of hidden layers for soaking temperature step.

\begin{tabular}{|c|c|c|c|c|c|}
\hline \multirow{2}{*}{ Hidden node number } & \multirow{2}{*}{ Model order $\left[\begin{array}{lll}n_{a} & n_{b} & n_{k}\end{array}\right]$} & \multirow{2}{*}{$\mathrm{LF}$} & \multicolumn{3}{|c|}{ Criterion } \\
\hline & & & AIC & BIC & MDL \\
\hline \multirow{5}{*}{5} & {$\left[\begin{array}{lll}2 & 1 & 9\end{array}\right]$} & $1.5 e-05$ & -5.7613 & -5.7314 & -5.6865 \\
\hline & {$\left[\begin{array}{lll}2 & 2 & 6\end{array}\right]$} & $7.2 e-04$ & -1.9165 & -1.8766 & -1.8167 \\
\hline & {$\left[\begin{array}{lll}2 & 3 & 8\end{array}\right]$} & $4.4 e-07$ & -9.3200 & -9.2701 & -9.1952 \\
\hline & {$\left[\begin{array}{lll}3 & 1 & 8\end{array}\right]$} & $6.7 e-05$ & -4.2955 & -4.2556 & -4.1957 \\
\hline & {$\left[\begin{array}{lll}3 & 2 & 8\end{array}\right]$} & $5.0 e-07$ & -9.1794 & -9.1295 & -9.0546 \\
\hline \multirow{5}{*}{10} & {$\left[\begin{array}{lll}2 & 1 & 6\end{array}\right]$} & $4.2 e-05$ & -4.7765 & -4.7466 & -4.7016 \\
\hline & {$\left[\begin{array}{lll}2 & 2 & 9\end{array}\right]$} & $6.9 e-07$ & -8.8683 & -8.8283 & -8.7684 \\
\hline & {$\left[\begin{array}{lll}2 & 3 & 8\end{array}\right]$} & $1.8 e-05$ & -5.6130 & -5.5631 & -5.4882 \\
\hline & {$\left[\begin{array}{lll}3 & 1 & 7\end{array}\right]$} & $8.6 e-02$ & 2.8603 & 2.9002 & 2.9601 \\
\hline & {$\left[\begin{array}{lll}3 & 2 & 3\end{array}\right]$} & $2.1 e-06$ & -7.7598 & -7.7099 & -7.6350 \\
\hline \multirow{5}{*}{15} & {$\left[\begin{array}{lll}2 & 1 & 9\end{array}\right]$} & $6.7 e-06$ & -6.5927 & -6.5627 & -6.5178 \\
\hline & {$\left[\begin{array}{lll}2 & 2 & 7\end{array}\right]$} & $3.5 e-04$ & -2.6251 & -2.5852 & -2.5252 \\
\hline & {$\left[\begin{array}{lll}2 & 3 & 8\end{array}\right]$} & $2.3 e-04$ & -3.0369 & -2.9870 & -2.9121 \\
\hline & {$\left[\begin{array}{lll}3 & 1 & 9\end{array}\right]$} & $6.1 e-06$ & -6.6938 & -6.6539 & -6.5940 \\
\hline & {$\left[\begin{array}{lll}3 & 3 & 3\end{array}\right]$} & $2.5 e-06$ & -7.5759 & -7.5260 & -7.4511 \\
\hline
\end{tabular}




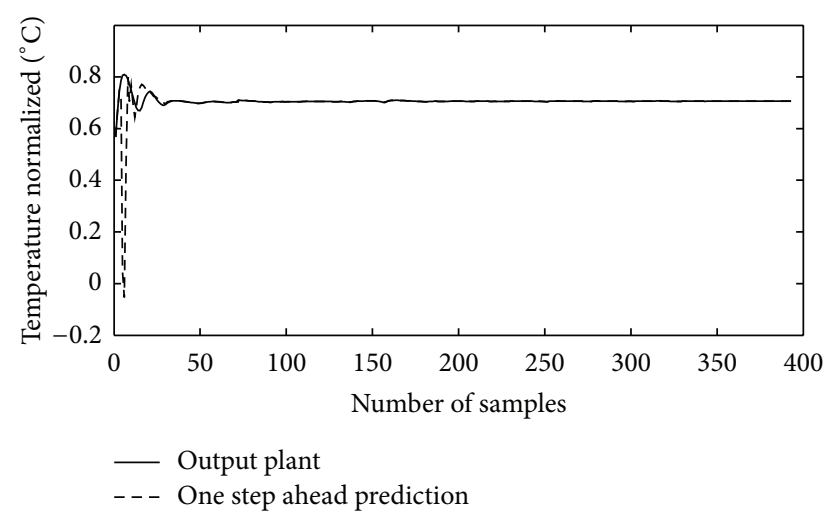

FIGURE 6: Output plant and one-step ahead prediction for constant heating rate step.

and MDL give a clear indication of a particular model; the interpretation of the results of these criteria clearly provides further support for the above choice of a model structure demonstrated by the loss function.

4.2. Simulation Result. The identification process was performed using the model structures that have been previously selected for the constant heating rate and soaking temperature steps.

In order to train NARX neural network model the dataset has to be normalized. Normalization implies that all values from the dataset should take values in the range from 0 to 1 . For that purpose the following formula would be used:

$$
X_{i, 0 \text { to } 1}=\frac{X_{i}-X_{\min }}{X_{\max }-X_{\min }},
$$

where, $X_{i}$ is each data point, $X_{\min }$ is the minima among all the data points, and $X_{\max }$ is the maxima among all the data points in the dataset.

Figure 6 shows the difference between the experimental output and those simulated parameters of the NARX neural network model [ $\left[\begin{array}{lll}2 & 1 & 7\end{array}\right]$ for constant heating rate step. Analyzing these figures, it appears that NARX models [l $\left.\begin{array}{lll}2 & 1 & 7\end{array}\right]$ have acceptable performance because it is able to correctly identify the dynamics of the constant heating rate step in the furnace with normalized sum of square error $1.9 e-03$. Relatively large prediction errors at the initial stage of the process as shown in Figure 7 caused by the inertia properties of the furnace at the beginning of the heating step.

Figure 8 shows the difference between the experimental output and those simulated parameters of the NARX neural network model $\left[\begin{array}{lll}2 & 3 & 8\end{array}\right]$ for soaking temperature step. Analyzing this figure, it emerges that the NARX model $\left[\begin{array}{lll}2 & 3 & 8\end{array}\right]$ ensures satisfactory performances as it is indeed able to correctly identify the dynamics of the soaking temperature step in the furnace with normalized sum square error $6.3859 e-08$. Figure 9 shows the prediction error for soaking temperature step in the furnace. The main advantage of the proposed neural approach consists in the natural ability of neural networks in modeling nonlinear dynamics in a fast and simple way and in the possibility to address the process

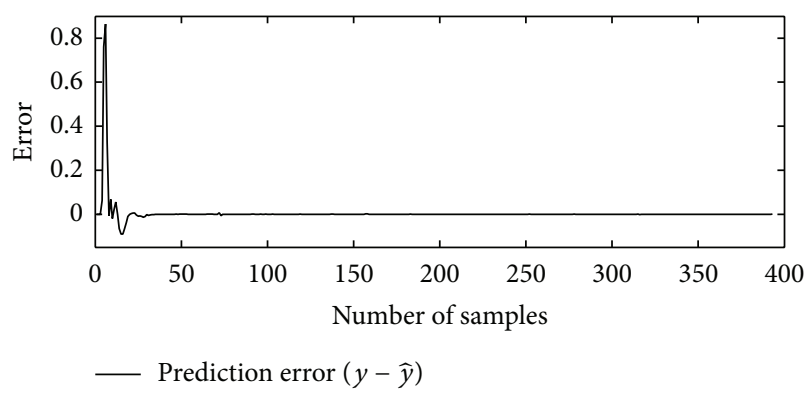

Figure 7: Prediction error for constant heating rate step.

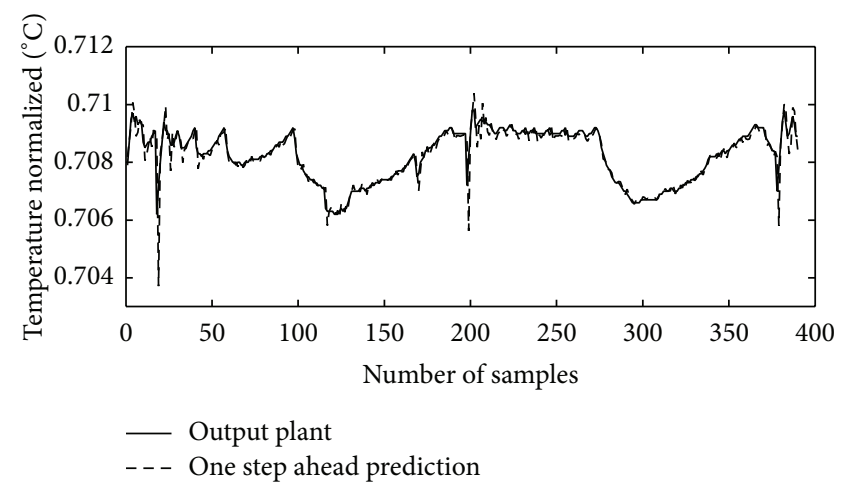

FIgURE 8: Output plant and one-step ahead prediction for soaking temperature step.

to be modeled as an input-output black box, with little or no mathematical information on the system.

In order to validate the identified model, it is necessary to evaluate the properties of the errors that affect the prediction of the outputs of the model, which can be defined as the differences between experimental and simulated time series. In general, the characteristics of the error are considered satisfactory when the error behaves as white noise; that is, it has a zero mean and the components are uncorrelated $[19,20]$. In fact, if both these conditions are satisfied, it means that the identified model has captured the deterministic part of the system dynamics, which is, therefore, accurately modeled. To this aim, it is necessary to verify that the autocorrelation function of the normalized error $\varepsilon(t)$, namely, $\phi \varepsilon \varepsilon(\tau)$, assumes the values 1 for $t=0$ and 0 elsewhere; in other words, it is required that the function behaves as an impulse. This autocorrelation is defined as follows [20, 21]:

$$
\phi \varepsilon \varepsilon(\tau)=E[\varepsilon(t-\tau) \varepsilon(t)]=\delta(\tau) \nabla \tau,
$$

where $\varepsilon$ is the model residual. $E(X)$ is the expected value of $X ; \tau$ is the lag. This condition is, of course, ideal and in practice it is sufficient to verify that $\phi \varepsilon \varepsilon(\tau)$ must remain inside range $\pm 1.96 / \sqrt{ } N$, with $N$ being the number of testing data on which $\phi \varepsilon \varepsilon(\tau)$ is calculated. Rankovic and Nikolic [8] proposed also tests for looking into the cross-correlation among model residuals and inputs. This cross-correlation is defined by the following equation:

$$
\phi u \varepsilon(\tau)=E[u(t-\tau) \varepsilon(t)]=0 \nabla \tau .
$$




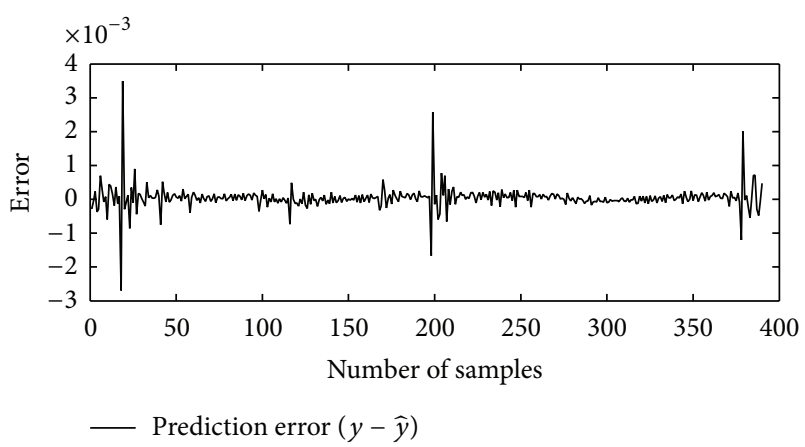

FIGURE 9: Prediction error for soaking temperature step.

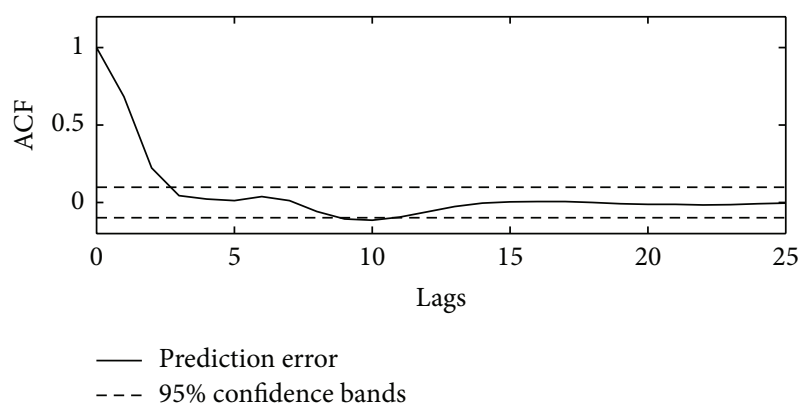

FIGURE 10: Autocorrelation function of prediction error for constant heating rate step.

To implement these tests, $u$ and $\varepsilon$ are normalized to give a zero mean sequence of unit variance. The sampled crossvalidation function between two such data sequences $u(t)$ and $\varepsilon(t)$ is then calculated as

$$
\phi u \varepsilon(\tau)=\frac{\sum_{t=1}^{N-\tau} u(t) \varepsilon(t+\tau)}{\left[\sum_{t=1}^{N} u^{2}(t) \sum_{t=1}^{N} \varepsilon^{2}(t)\right]^{1 / 2}} .
$$

If (10) and (11) are satisfied then the model residuals are a random sequence and are not predictable from inputs and, hence, the model will be considered adequate. These correlations based tests are used here to validate the neural network model. The results are presented in Figure 10 to Figure 13 for the constant heating rate and soaking temperature steps, respectively. In these plots, the dash dot lines are the 95\% confidence bands.

As shown in Figures 10 and 11, the autocorrelation of the NARX neural model for constant heating rate and soaking temperature steps, most points are inside the $95 \%$ confidence bands.

Figures 12 and 13 shows that the evolution of the crosscorrelation of the NARX model, most points are within the 95\% confidence band for constant heating rate and soaking step of the sintering process. In addition, the NARX crosscorrelation is low. This explains the independence of the residual signal from the input one. Therefore, this model is considered a reliable one for describing the dynamic behavior of the process without any significant loss of accuracy appropriate to the complexity of the model. This validation phase is used with the neural weights found in the training

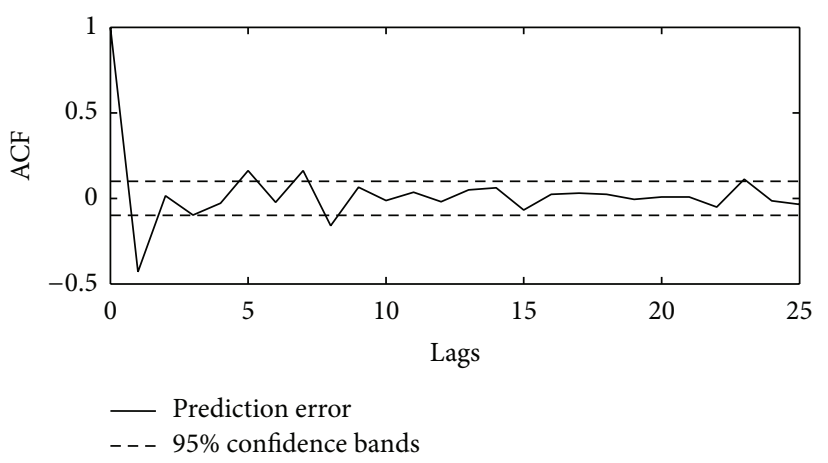

FIGURE 11: Autocorrelation function of prediction error for soaking temperature step.

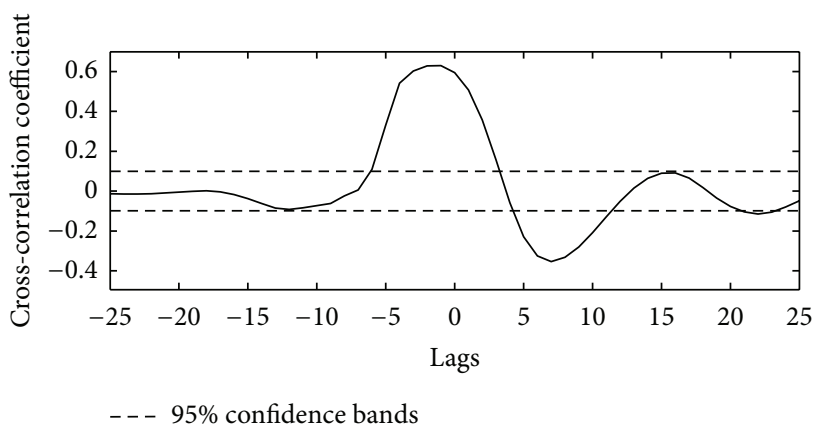

FIGURE 12: Cross-correlation function of $u 1$ and prediction error for constant heating rate step.

phase. There is a good agreement between the learned neural model and the experiment in the validation phase.

\section{Conclusion}

This work aims to identify the process dynamics by means of an NARX model. The identification of the system dynamics by means of input-output experimental measurements provides a useful solution for the formulation of a reliable model. This paper aimed at identifying the dynamics of a process of sintering temperature in order to provide reliable predictions. The identification of the system was performed by means of the NARX approach implemented using a neural network. In this case, the results showed that the model is able to give satisfactory descriptions of the experimental data with normalized sum of square errors $1.9 e-03$ for constant heating rate step and $6.3859 e-08$ for soaking temperature step. Although the predictive capability of the models is limited to a few steps ahead and varies with the variable considered, the time for which satisfactory predictions were achieved is sufficient for the implementation of the NARX neural models control with offline learning using recorded data and can be improved by implementing an online learning method as well as other variables that affect the temperature of the furnace for more complex control schemes to improve the performance. 


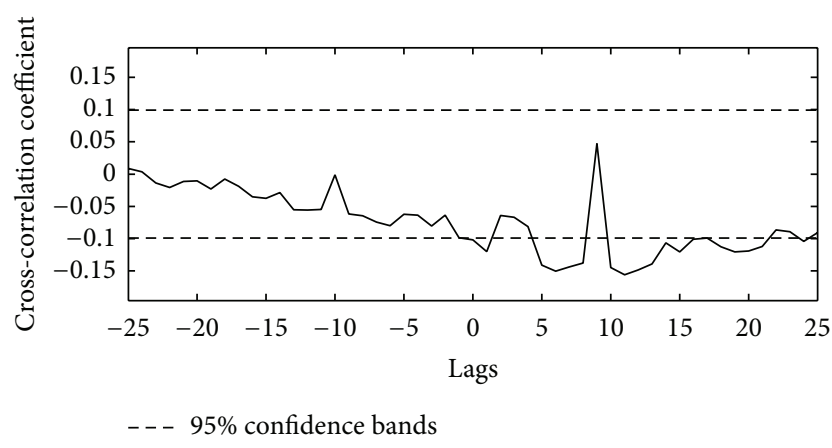

FIGURE 13: Cross-correlation function of $u 1$ and prediction error soaking temperature step.

\section{Conflict of Interests}

The work presented in this paper was partly funded by the Ministry of Science and Technology of Indonesia and National Nuclear Agency of Indonesia. The authors would like to thank them for their support in this important work. The terms of this arrangement have been reviewed and approved by the University of Indonesia in accordance with its policy on objectivity in research.

\section{References}

[1] H. Al-Hiary, M. Braik, A. Sheta, and A. Ayesh, "Identification of a chemical process reactor using soft computing techniques," in Proceedings of the IEEE International Conference on Fuzzy Systems (FUZZ '08), pp. 845-853, June 2008.

[2] K. Valarmathi, D. Devaraj, and T. K. Radhakrishnan, "Intelligent techniques for system identification and controller tuning in $\mathrm{pH}$ process," Brazilian Journal of Chemical Engineering, vol. 26, no. 1, pp. 99-111, 2009.

[3] A. K. Jain, J. Mao, and K. M. Mohiuddin, "Artificial neural networks: a tutorial," IEEE Computer. Special Issue on Neural Computing, vol. 29, no. 3, pp. 31-44, 1996.

[4] H. Uchida Frausto and J. G. Pieters, "Modelling greenhouse temperature using system identification by means of neural networks," Neurocomputing, vol. 56, pp. 423-428, 2004.

[5] T. R. Biyanto, M. Ramasamy, and H. Zabiri, "Modeling heat exchanger using neural networks," in Proceedings of the International Conference on Intelligent and Advanced Systems (ICIAS '07), pp. 120-124, November 2007.

[6] Y. Lee and T. L. Chang, "Application of NARX neural networks in thermal dynamics identification of a pulsating heat pipe," Energy Conversion and Management, vol. 50, no. 4, pp. 10691078, 2009.

[7] M. Rajalakshmi, G. Saravanakumar, and C. Karthik, "Nonlinear identification of $\mathrm{pH}$ processes by using NNARX model," in Proceedings of the International Conference on Computing and Control Engineering (ICCCE '12), Coimbatore Institute of Information Technology, 2012.

[8] V. M. Rankovic and I. Z. Nikolic, "Identification of nonlinear models with feedforward neural networks and digital recurrent network," FME Transactions, vol. 36, pp. 87-92, 2008.

[9] A. Hosovsky and J. Mizak, "Dynamic modeling of biomass fired boiler emissions," Journal of Applied Science in the Thermodynamics and Fluid Mechanics, vol. 4, no. 1, pp. 1-6, 2010.
[10] C. Alippi and V. Piuri, "Experimental neural networks for prediction and identification," IEEE Transactions on Instrumentation and Measurement, vol. 45, no. 2, pp. 670-676, 1996.

[11] J. I. Canelon, L. S. Shieh, and N. B. Karayiannis, "A new approach for neural control of nonlinear discrete dynamic systems," Information Sciences, vol. 174, no. 3-4, pp. 177-196, 2005.

[12] A. D. Nira, Sintering Furnace Manual, Indonesia Project, No. IND-700-00-Q-0498pp, pp. 50-66, 1986.

[13] L. Ljung, System Identification: Theory for the User, Prentice Hall, Upper Saddle River, NJ, USA, 1999.

[14] T. Soderstrom and P. Stoica, System Identification, Prentice Hall, London, UK, 1989.

[15] P. M. J. van den Hof and R. J. P. Schrama, "Identification and control-closed-loop issues," Automatica, vol. 31, no. 12, pp. 1751-1770, 1995.

[16] R. A. Eek, J. A. Both, and P. M. J. van den Hof, "Closed-loop identification of a continuous crystallization process," AIChE Journal, vol. 42, no. 3, pp. 767-776, 1996.

[17] U. Forssell and L. Ljung, "Closed-loop identification revisited," Automatica, vol. 35, no. 7, pp. 1215-1241, 1999.

[18] A. Esmaili, J. F. MacGregor, and P. A. Taylor, "Direct and twostep methods for closed-loop identification: a comparison of asymptotic and finite data set performance," Journal of Process Control, vol. 10, no. 6, pp. 525-537, 2000.

[19] L. Cammarata, A. Fichera, and A. Pagano, "Neural prediction of combustion instability," Applied Energy, vol. 72, no. 2, pp. 513$528,2002$.

[20] S. A. Billings and W. S. F. Voon, "Correlation based validity tests for nonlinear models," International Journal of Control, vol. 44, no. 1, pp. 235-244, 1996.

[21] J. Zhang and J. Morris, "Process modelling and fault diagnosis using fuzzy neural networks," Fuzzy Sets and Systems, vol. 79, no. 1, pp. 127-140, 1996. 


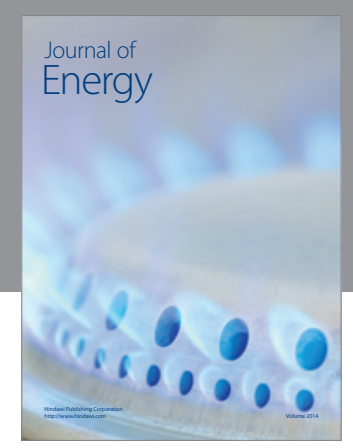

Journal of

Industrial Engineering
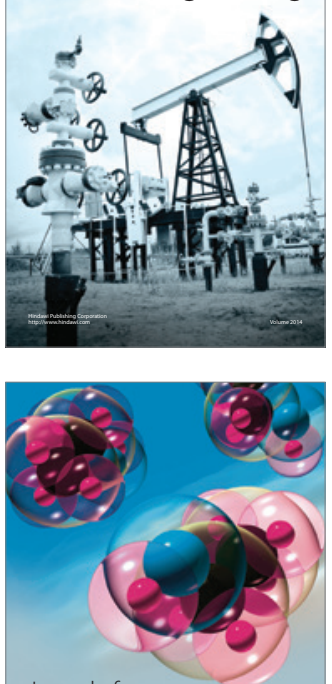

Fuels
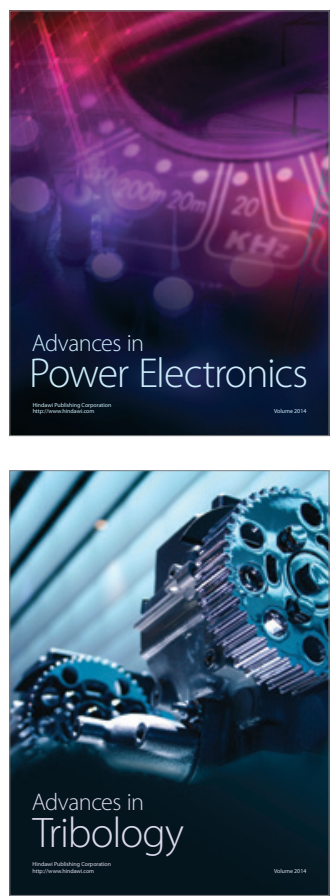

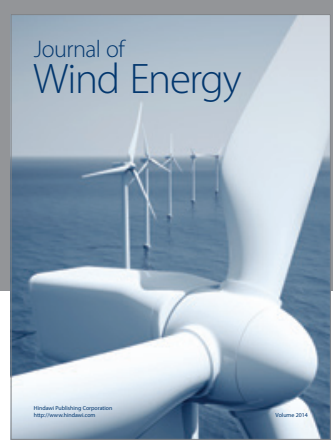

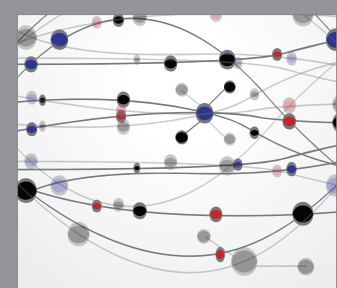

The Scientific World Journal

Submit your manuscripts at http://www.hindawi.com

Journal of

Structures
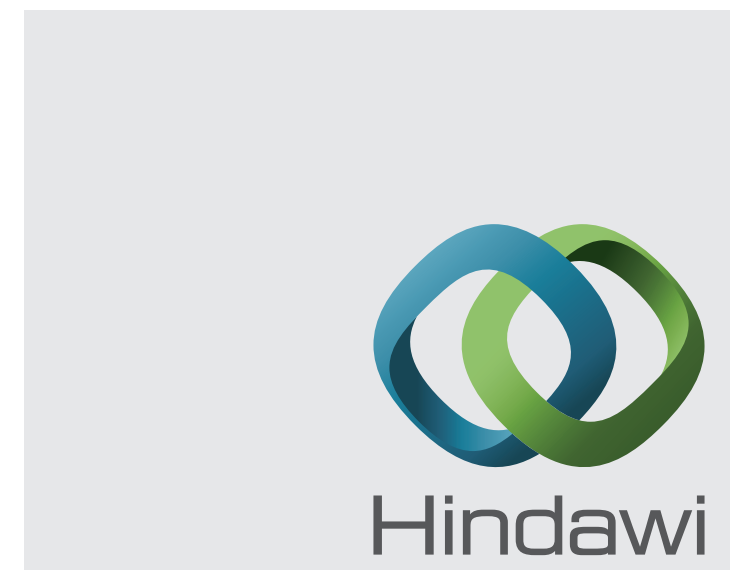

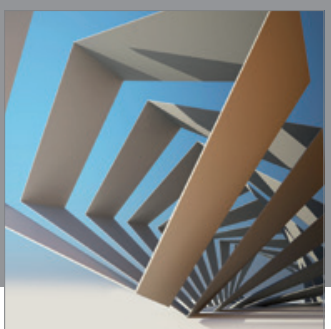

Rotating

Machinery
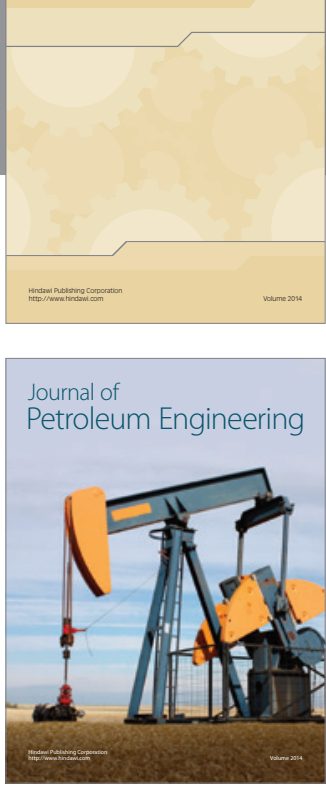

Journal of

Solar Energy
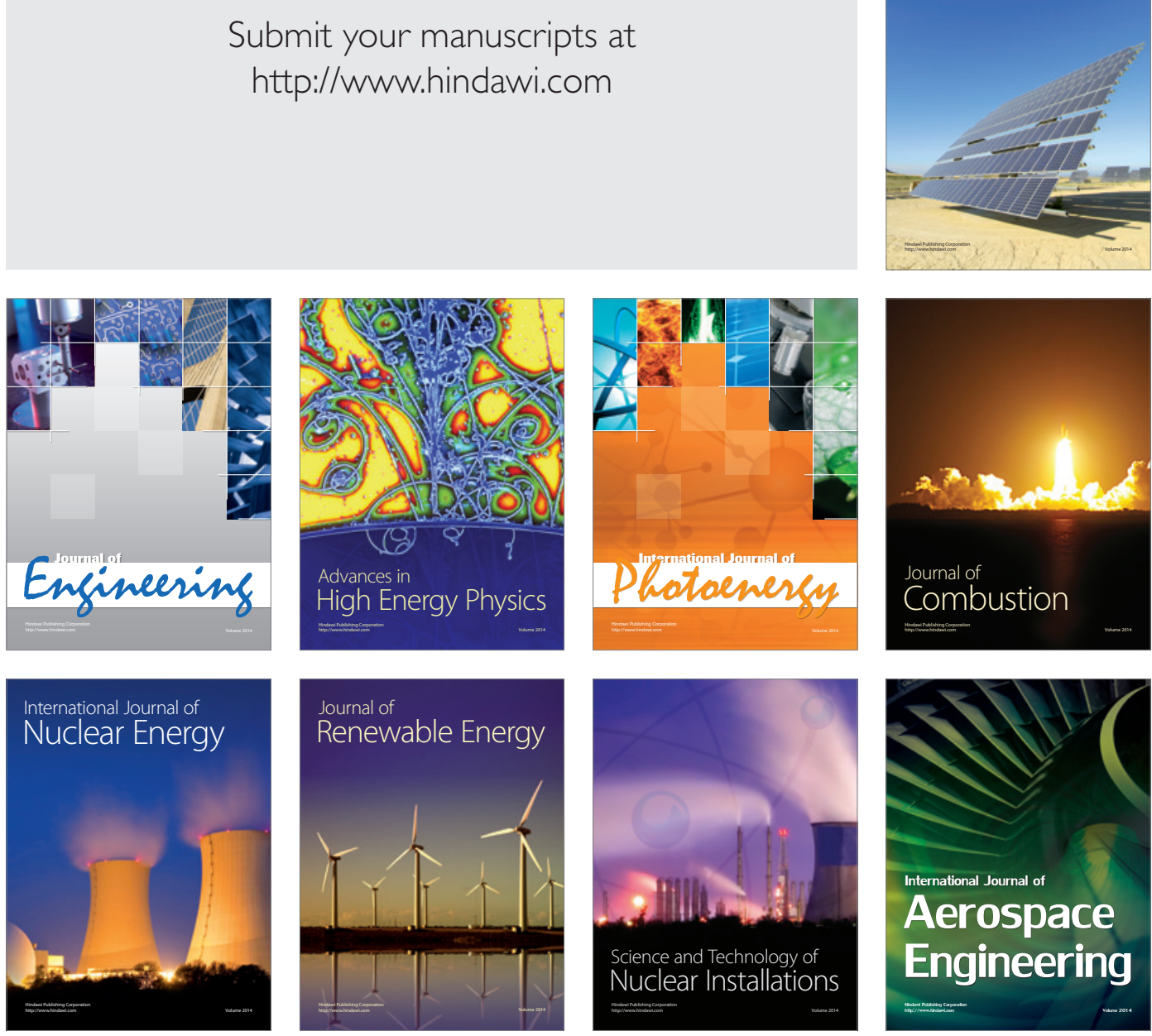